Acta bot. bras. 21(3): 521-529. 2007

\title{
Diatomáceas indicadoras de paleoambientes do Quaternário de Dois Irmãos, Recife, PE, Brasil
}

\author{
Giane Soares de Souza ${ }^{1}$, Maria Luise Koening ${ }^{2,5}$, Enide Eskinazi Leça ${ }^{3} \mathrm{e}$ \\ Maria de Pompéia Correia de A. Coêlho ${ }^{4}$
}

Recebido em 10/11/2005. Aceito em 21/11/2006

\begin{abstract}
RESUMO - (Diatomáceas indicadoras de paleoambientes do Quaternário de Dois Irmãos, Recife, PE, Brasil). O trabalho apresenta o levantamento da diatomoflórula identificada em sedimentos do Quaternário da Lagoa das Diatomáceas (Dois Irmãos, Recife), baseado em um testemunho de sondagem com 4,5 m, tendo sido analisadas amostras com intervalos de $10 \mathrm{em} 10 \mathrm{~cm}$. Foram identificados 46 táxons, distribuídos em 19 gêneros, 40 espécies e seis variedades. A coluna estratigráfica esteve composta por gêneros epífitos (Actinella, Amphora, Cocconeis Eunotia, Fragilaria, Frustulia, Gomphonema e Rhopalodia), bentônicos (Navicula, Neidium, Nitzschia e Surirella) e planctônicos (Aulacoseira, Cyclotella e Skeletonema). A maioria das espécies é oligoalóbia e litoral, destacando-se em termos de frequiência e abundância: Actinella brasiliensis, Anomoeoneis serians, Eunotia pectinalis, Frustulia rhomboides, sugerindo uma deposição de sedimentos em ambiente limnético. O predomínio de espécies epífitas em determinadas profundidades confirma a presença de macrófitas durante uma parte do período de deposição. Das espécies identificadas sete são consideradas de ambiente marinho: Cocconeis heteroidea, Cocconeis scutellum, Diploneis decipiens, Nitzschia scalaris, Nitzschia sigma, Skeletonema costatum e Surirella heideni as quais ocorreram de forma esporádica.
\end{abstract}

Palavras-chave: diatomáceas, Quaternário, fóssil, estratigrafia

ABSTRACT - (Diatoms as indicators of paleoenvironments during the Quaternary at Dois Irmãos, Recife, Pernambuco State, Brazil). A study of fossil diatoms from Lagoa das Diatomáceas (Dois Irmãos, Recife) was carried out based on a 4.5-meter-long core sample. Samples were analyzed at $10 \mathrm{~cm}$ intervals. A total of 46 taxa were identified in 19 genera, 40 species and six varieties. The stratigraphic column was composed of epiphytic (Actinella, Amphora, Cocconeis Eunotia, Fragilaria, Frustulia, Gomphonema and Rhopalodia), benthic (Navicula, Neidium, Nitzschia, Surirella), and planktonic (Aulacoseira, Cyclotella and Skeletonema) genera. Actinella brasiliensis, Anomoeoneis serians, Eunotia pectinalis and Frustulia rhomboides are oligohalobe, littoral species with high frequency and abundance, which suggests sediment deposition in a limnetic environment. Epiphyte dominance at certain depths confirms the presence of macrophytes during the deposition period. Only seven of the identified species are from a marine environment: Cocconeis heteroidea, Cocconeis scutellum, Diploneis decipiens, Nitzschia scalaris, Nitzschia sigma, Skeletonema costatum and Surirella heideni which occurred sporadically.

Key words: diatoms, Quaternary, fossil, stratigraphy

\section{Introdução}

As diatomáceas constituem um dos principais grupos na base da cadeia alimentar de todos os ecossistemas aquáticos, estimando-se que a produção dessas microalgas no ambiente marinho esteja em torno de $2 \times 10{ }^{13} \mathrm{~kg} \mathrm{C} /$ ano, cuja produção representa 20 a 25\% da produção primária mundial (Moreno et al. 1996).

São algas ubíquas, apresentam uma ampla distribuição, sendo encontradas desde ambientes aquáticos, terrestres ou subaéreos, podendo viver fixas como epífitas em macroalgas e fanerógamas marinhas, flutuando livremente ou sobre substrato orgânico (Bold \& Wynne 1985). São encontradas em grandes quantidades no plâncton marinho e de água doce, onde podem formar densos lençóis flutuantes (Cooper 1999).

A presença de diatomáceas em registros fósseis deve-se à natureza resistente de sua parede celular que é composta de sílica, facilitando a formação de depósitos silicosos denominados diatomitos. Tais depósitos são encontrados nos oceanos ou em

\footnotetext{
1 Parte da Dissertação de Mestrado da primeira Autora - Programa de Pós-Graduação em Biologia Vegetal da Universidade Federal de Pernambuco (gianesoares@yahoo.com.br)

2 Universidade Federal de Pernambuco, Departamento de Oceanografia, Av. Arquitetura s/n, Cidade Universitária, 50670-901 Recife, PE, Brasil (koening@ufpe.Br)

3 Universidade Federal Rural de Pernambuco, Departamento de Biologia, Rua Dom Manoel de Medeiros s/n, Dois Irmãos, 52171-900 Recife, PE, Brasil (enideleca@terra.com.br)

4 Sociedade Nordestina de Ecologia (mariadepompeia@bol.com.br)

5 Autor para correspondência: koening@ufpe.br
} 
ambientes limnéticos. A identificação das espécies pode ser utilizada também para interpretações paleoecológicas: determinar se os sedimentos são de origem marinha ou não, mostrar de que modo às características lacustres foram mudadas durante o período Quaternário, especialmente em relação a eutrofização antropogênica e acidificação, reconstruir os climas passados e as condições passadas nos oceanos e ecossistemas aquáticos (Ruiz-Moreno \& Carreño 1994; Yim \& Li 2000). Em alguns países, estes depósitos sedimentaram-se no período Cretáceo (Era Mesozóica) e, no Brasil, datam do período Quaternário (Era Cenozóica) (Moreira 1975).

O Quaternário caracteriza-se por profundas mudanças climáticas, assim como pelo surgimento do Homem. Mundialmente, os estudos referentes ao Quaternário são relativamente recentes e por isso alguns não atingiram sua completa maturidade e sistematização mais definida. No Brasil, a situação não é diferente e o país não apresenta uma literatura numerosa (Suguio 1999). Em Pernambuco, a pesquisa sobre diatomáceas fósseis é, ainda, pouco desenvolvida, merecendo destaque o trabalho pioneiro de Souza \& Abreu (1939) que estudaram depósitos do Rio Grande do Norte, Ceará e Pernambuco, e algumas observações fragmentárias de espécies ocorrentes em depósitos de Dois Irmãos, que foram apresentados por Moreira (1975). Por outro lado, as diatomáceas recentes marinhas e dulciaquíícolas do nordeste são bastante conhecidas, quanto à composição florística e aspectos quantitativos (Eskinazi-Leça et al. 1996), o que facilita uma comparação florística entre os registros atuais e os encontrados em sedimentos do quaternário.

Para um maior conhecimento das diatomáceas fósseis de Pernambuco, foi realizado um levantamento florístico das espécies observadas em um testemunho de sondagem retirado da lagoa das diatomáceas, localizada no bairro de Dois Irmãos, Recife, PE.

\section{Material e métodos}

O material para estudo foi obtido na Lagoa das Diatomáceas $\left(08^{\circ} 01104,911\right.$ Lat. S. e 3456'56,5" Long. W.) - bairro de Dois Irmãos, que se caracteriza como sendo uma antiga lagoa de restinga, hoje transformada em uma baixada alagada e preenchida por um material turfoso. A área também apresenta depósitos Quaternários, que são constituídos por sedimentos terrígenos (areias, argilas e conglomerados). Nas planícies fluviolacustres que caracterizam a área estudada, dominam areias finas, siltes, argilas, massas diatomáceas, sedimentos turfáceos e depósitos de mangue (CPRM 2001).

A Lagoa das Diatomáceas está localizada dentro do núcleo urbano do Recife, PE (Reserva Ecológica de Dois Irmãos), na chamada Planície do Recife, em torno da qual se elevam ao norte, ao oeste e ao sul, em semicírculo quase perfeito, as Colinas da Formação Barreiras. A leste tem-se o Oceano Atlântico. Ao longo da linha litorânea, a Planície do Recife tem a extensão de cerca de $15 \mathrm{~km}$, e na direção oeste, uma extensão de, aproximadamente, $14 \mathrm{~km}$ (Coutinho et al. 1998; CPRH 2001). As variações do nível relativo do mar, de pequena amplitude e curta duração, foram importantes no desenvolvimento das porções mais recentes das Planícies Costeiras, inclusive na instalação dos depósitos fluvio-lagunares, os quais podem intercalar sedimentos tipicamente lagunares, ricos em conchas, com sedimentos de água doce depositados em lagoas, brejos e pântanos. Na subsuperfície são encontradas camadas de argilas moles, diatomitos e turfas, respectivamente oriundos desses ambientes. Assim, a evolução das Planícies Costeiras, inclusive da Planície do Recife, está diretamente relacionada às variações do nível do mar que ocorreram durante o Período Quaternário, tendo Coutinho et al. (1998) considerado as seguintes fases evolutivas: a) a primeira fase iniciada pela deposição da Formação Barreiras, tendo acontecido no final de Plioceno, depositando-se até a atual plataforma continental submersa; b) a segunda fase, representada pela chamada Máxima Transgressão, o nível do mar elevado, erodiu parte da Formação Barreiras, esculpindo falésias; c) a terceira fase é a da deposição pós-Barreiras, conseqüência da regressão, que se sucedeu após a fase anterior. Esta fase permitiu a deposição dos sedimentos continentais em forma de leques aluviais acumulados no sopé das falésias; d) na quarta fase aconteceu a chamada Penúltima Transgressão, na qual os depósitos da fase anterior foram retrabalhados e cobertos; e) na quinta fase houve a regressão subseqüente com a formação dos Terraços Marinhos Pleistocênicos, situados atualmente numa altitude de 6 a $10 \mathrm{~m}$. Nesta época, possivelmente o rio Capibaribe mudou de curso, passando da direção S 30 E para N 40 E, na altura de Dois Irmãos, ocasionando a erosão de parte do terraço marinho pleistocênico e favorecendo a instalação, na planície, de zonas de pântanos e/ou lagunas; d) a sexta fase é marcada pela Última Transgressão, tendo ocorrido o afogamento das planícies costeiras pleistocênicas e a estabilidade do nível do mar, que se seguiu ao máximo transgressivo de 5.100 anos A.P, permitiu a formação dos Terraços 
Marinhos Holocênicos.

O testemunho foi datado pelo método do ${ }^{14} \mathrm{C}$, pelo Institut de Recherche Pour le Developpment (ORSTOM) e a idade mais antiga, na base do tetemunho, foi de 7.000 A.P.

O testemunho de sondagem apresenta 14 intervalos, assim caracterizados: o intervalo superior, entre 0 e $15 \mathrm{~cm}$, apresenta cor marrom acinzentado, com textura argilo-arenoso, pedaços de material vegetal carbonizados e raízes de vegetação atual; o intervalo seguinte, entre, 15 a $31 \mathrm{~cm}$, de cor cinza, textura argilosa e alguns pedaços de carvão; o intervalo entre 31 a $53 \mathrm{~cm}$ apresenta cor marrom acinzentada, textura argilo-arenosa, pedaços de vegetais, carvão e nódulos de argila endurecidos; o intervalo seguinte, entre 53 e $81 \mathrm{~cm}$, apresenta cor marrom muito escura, textura orgânica e numerosos pedaços de vegetais de dimensões de até $10 \mathrm{~cm}$, pedaços de madeira situados entre 70 e $80 \mathrm{~cm}$; o intervalo entre 81 e $95 \mathrm{~cm}$ apresenta cor marrom escuro, textura orgânica e alguns pedaços de vegetais com dimensões centimétricas com cerca de $85 \mathrm{~cm}$; o intervalo entre 95 e $107 \mathrm{~cm}$, apresenta cor marrom acinzentado escuro, textura orgânica mais fina do que o material vegetal da camada acima, pedaços de madeira situados entre 104 e $106 \mathrm{~cm}$; o intervalo entre 107 e $121 \mathrm{~cm}$ apresenta cor marrom acinzentado muito escuro, textura claramente síltica e matéria orgânica fina; intervalo entre $121 \mathrm{e} 176 \mathrm{~cm}$, cor marrom acinzentado muito escuro, textura argilo-arenoso muito orgânico, pedaços de vegetais de grande tamanho aumentando do ápice para a base, numerosos pedaços de carvão de tamanho centimétrico e madeiras situados entre 130 e $169 \mathrm{~cm}$; o intervalo entre 176 e $252 \mathrm{~cm}$ apresenta cor marrom acinzentado muito escuro, textura orgânica, restos vegetais de grande dimensão (folhas, raízes) e numerosos pedaços de carvão em toda a sequiência e madeiras entre 195 e $239 \mathrm{~cm}$, o intervalo seguinte, 252 a $310 \mathrm{~cm}$, apresenta cor marrom acinzentado muito escuro, textura orgânica, numerosos pedaços de carvão espaçados, pedaços de madeira em toda a sequiência e madeira situada entre 263,5 e 277; o intervalo entre 310 e $319 \mathrm{~cm}$ apresenta cor cinza escuro, textura orgânica e pedaços de carvão espaçados; o intervalo entre 319 e $384 \mathrm{~cm}$ apresenta cor marrom escuro, textura orgânica, pedaços de madeira, restos de vegetais (folhas) sedimentadas horizontalmente, pedaços de carvão espaçados e madeiras situadas entre 358 e $359 \mathrm{~cm}$; o intervalo entre $384 \mathrm{e} 412 \mathrm{~cm}$ apresenta cor cinza, textura argilosa muito orgânica, pedaços de carvão, folhas e concentração de restos vegetais nos 5 últimos centímetros; o intervalo basal, compreendido entre 412 e $454 \mathrm{~cm}$ apresenta cor marrom acinzentado, textura arenosa muito orgânica até $418 \mathrm{~cm}$, bolsões de areia, grandes, entre 442 e $445 \mathrm{~cm}$, menores mais para o topo, pedaços de carvão dispersos situados entre 413 e $444 \mathrm{~cm}$, e coletados para datação do ${ }^{14} \mathrm{C}$.

$\mathrm{Na}$ coleta foi utilizado um tripé com tubo de irrigação de alumínio (vibro testemunhador) desenvolvido segundo metodologia de Martin \& Flexor (1991). Após a coleta, o tubo (testemunho de sondagem) teve suas extremidades vedadas com tampas plásticas e fita adesiva, marcado no sentido base-topo e depositado no Departamento de Biologia - Área de Ecologia da Universidade Federal Rural de Pernambuco. O mesmo foi denominado como PE - 3/92, apresentando profundidade de $4,5 \mathrm{~m}$. Posteriormente, o tubo foi devidamente cortado com uma serra elétrica e o sedimento separado em fatias transversais de $1 \mathrm{~cm}$ cada, exceto na primeira sub-amostra $(0-2,5 \mathrm{~cm})$, sendo estas, por sua vez, divididas em seis amostras que serviram para diversas análises paleontológicas. As amostras utilizadas para a análise das diatomáceas tiveram intervalo de $10 \mathrm{~cm}$ entre elas, totalizando 46 amostras.

Foram preparadas lâminas de microalgas de acordo com o método descrito por Müller-Melcher \& Ferrando (1956) e feitas às identificações qualitativa e quantitativa de acordo com bibliografia especializada. As características ecológicas dos taxa foram baseadas na literatura, como também no trabalho de Moreira Filho et al. (1999) e o enquadramento taxonômico foi baseado no Sistema de Classificação de Simonsen (1979).

Foram calculadas as freqüências de ocorrência de acordo com o número de amostras onde cada táxon ocorreu (Mateucci \& Colma 1982) sendo adotadas as seguintes categorias: Muito freqüente $=>70 \%$ das amostras; Freqüente $=70 \% \mathrm{I}-40 \%$; Pouco Freqüente $=40 \% \mathrm{I}-20 \%$; Esporádica $=\leq 20 \%$.

A variação da abundância relativa dos táxons esteve baseada entre a densidade absoluta de uma espécie e o somatório das densidades absolutas de todas as espécies presentes em cada lamina, como uma forma de obtenção da representação quantitativa de cada táxon. Foi adotado o seguinte critério para o enquadramento dos táxons, segundo Lobo \& Leighton (1986): Dominante $=>50 \%$; Abundante $=50 \% \mathrm{~L}-30 \%$; Pouco Abundante $=30 \% \mathrm{~L}-10 \%$; Rara $=\leq 10 \%$.

\section{Resultados e discussão}

Foram identificados na análise estratigráfica 46 táxons, representados por 19 gêneros, 40 espécies e 
seis variedades (Tab. 1). A maioria dos táxons esteve distribuído na Ordem Pennales, com 91,31\% da diatomoflórula, estando a ordem representada por sete famílias: Naviculaceae (17 spp.), Eunotiaceae (9 spp.), (4 var.), Nitzschiaceae (5 spp., 2 var.), Achnanthaceae (2 spp.), Surirellaceae (2 spp.), Diatomaceae (1 sp.), Epithemiaceae (1 sp.), e destacando-se os gêneros Pinnularia (8 spp.) e Eunotia (8 spp. e 4 var.).

A Ordem Centrales compreendeu 8,69\% do total identificado e as espécies identificadas foram enquadradas na família Thalassiosiraceae (3 spp.). As Fig. 1 (A-K) e Fig. 2 (A-H) apresentam as espécies e variedades de diatomáceas mais representativas na coluna estratigráfica.

Particularmente para o nordeste brasileiro, são conhecidas algumas pesquisas que se referem a diatomáceas do Quaternário, destacando-se Souza \& Abreu (1939) que estudaram depósitos do Rio Grande do Norte, Ceará e Pernambuco, e Moreira (1975), que analisou três amostras de diatomitos do Quaternário de Pernambuco. Para o Estado da Bahia, Füstenberger (2001) identificou 38 táxons de diatomáceas, entre eles Anomoeoneis serians, Actinella brasiliensis e Frustulia rhomboides em testemunho de sondagem coletado no rio Icatu, com características semelhantes ao paleoambiente de Dois Irmãos.

A distribuição das espécies na coluna estratigráfica não foi uniforme, com uma maior deposição de diatomáceas nas camadas próximas ao topo $(0$ a $190 \mathrm{~cm}$ de profundidade), ocorrendo um declínio e até ausência na diatomoflórula, a partir da profundidade $190 \mathrm{~cm}$ incluindo as profundidades de 270, 290, 300, 310 a 330, 350 a 370, 390 a 410, 430 a 450 cm (Fig. 3). A não deposição de diatomáceas nos diferentes níveis estratigráficos foi também constatada por Moro \& Bicudo (1998), na lagoa Dourada, estado do Paraná, com a não deposição de diatomáceas entre 11,3 m e 9,9 m, ressaltando os autores que falhas na deposição de diatomáceas em sedimentos são registradas por vários autores atribuindo a diagêne, ou seja, a dissolução pósdeposicional das frústulas como a hipótese mais aceita.

No que se refere à abundância relativa (Fig. 4), quatro espécies destacaram-se em relação às demais: Actinella brasiliensis, Anomoeoneis serians, Eunotia pectinalis e Frustulia rhomboides, todas consideradas epífitas e bênticas, porém, nenhuma das espécies identificadas apresentou caráter dominante. Estas mesmas espécies já foram assinaladas em sedimentos quaternários do nordeste do Brasil por Souza \& Abreu (1939), Moreira (1975) e também em sedimentos do delta do Nilo (Zalat, 1995).

No testemunho de sondagem de Dois Irmãos a comunidade das diatomáceas caracterizou-se pela presença de espécies reconhecidamente de ambientes limnéticos, como os representantes de Actinella, Anomoeoneis, Eunotia, Frustulia e Pinnularia, além

Tabela 1. Espécies de diatomáceas identificadas na coluna estratigráfica coletada em sedimentos do Quaternário de Dois Irmãos, Recife, PE, Brasil.

\begin{tabular}{|c|c|}
\hline Famílias & Espécies/Variedades \\
\hline Thalassioraceae & $\begin{array}{l}\text { Cyclotella meneghiniana Kützing, Skeletonema costatum (Grév.) Cleve, Aulacoseira granulata (Ehr.) } \\
\text { Simonsen. }\end{array}$ \\
\hline Diatomaceae & Fragillaria sp. \\
\hline Eunotiaceae & $\begin{array}{l}\text { Actinella brasiliensis Grunow, Eunotia camelus Ehrenberg, Eunotia didyma Grunow var. didyma, Eunotia } \\
\text { didyma Grunow var. elongata Hustedt, Eunotia didyma Grunow var. jungulata Freguelli,Eunotia } \\
\text { femoribus (Patr.) Hustedt, Eunotia flexuosa (Bréb.) Kützing, Eunotia major (W. Sm.) Rabh. var. } \\
\text { emarginata A. Cleve, Eunotia pectinalis (Dill.), Eunotia trigibba Hustedt, Eunotia vumbae Choln., } \\
\text { Eunotia zygodon Ehrenberg, Eunotia sp. }\end{array}$ \\
\hline Achnanthaceae & Cocconeis scutellum Ehrenberg, Cocconeis heteroidea Kützing \\
\hline Epithemiaceae & Rhopalodia musculus (Kütz.) Otto Müller \\
\hline Naviculaceae & $\begin{array}{l}\text { Amphora } \mathrm{sp}_{\cdot_{1}} \text {, Amphora } \mathrm{sp}_{._{2}} \text {, Anomoeoneis serians (Bréb. Ex Kütz.) Cleve, Diploneis decipiens Cleve, } \\
\text { Frustulia rhomboides (Ehr.) Det., Gomphonema gracile Ehrenberg, Navicula } \mathrm{sp}_{\cdot}, \text {, Navicula } \mathrm{sp}_{.2} \text {, Neidium } \\
\text { affine (Ehr.) Pfitzer, Pinnularia abaujensis Pantocsek, Pinnularia biceps Gregory, Pinnularia maior } \\
\text { (Kütz.) Rabh., Pinnularia nobilis Ehrenberg, Pinnularia stauroptera (Grun.) Rabenhorst, Pinnularia } \\
\text { stomatophoroides Mayer, Pinnularia } \mathrm{sp}_{._{1}} \text {, Pinnularia } \mathrm{sp}_{._{2}}\end{array}$ \\
\hline Nitzschiaceae & $\begin{array}{l}\text { Hantzschia amphyozys (Ehr.) Grun. var. aequalis Cleve-Euler, Hantzschia amphyozys (Ehr.) Grun. var. } \\
\text { capitata Otto Müller, Nizschia scalaris (Ehr.) Wm. Smith, Nitzschia sigma (Kütz.) Wm. Smith, Nitzschia } \\
\text { sigmoidea } \mathrm{Wm} \text {. Smith, Nitzschia praelonga } \mathrm{Cl} \text {. Grunow, Nitzschia } \text { sp. }\end{array}$ \\
\hline Surirellaceae & Surirella heideni Hustedt, Surirella sp. \\
\hline
\end{tabular}




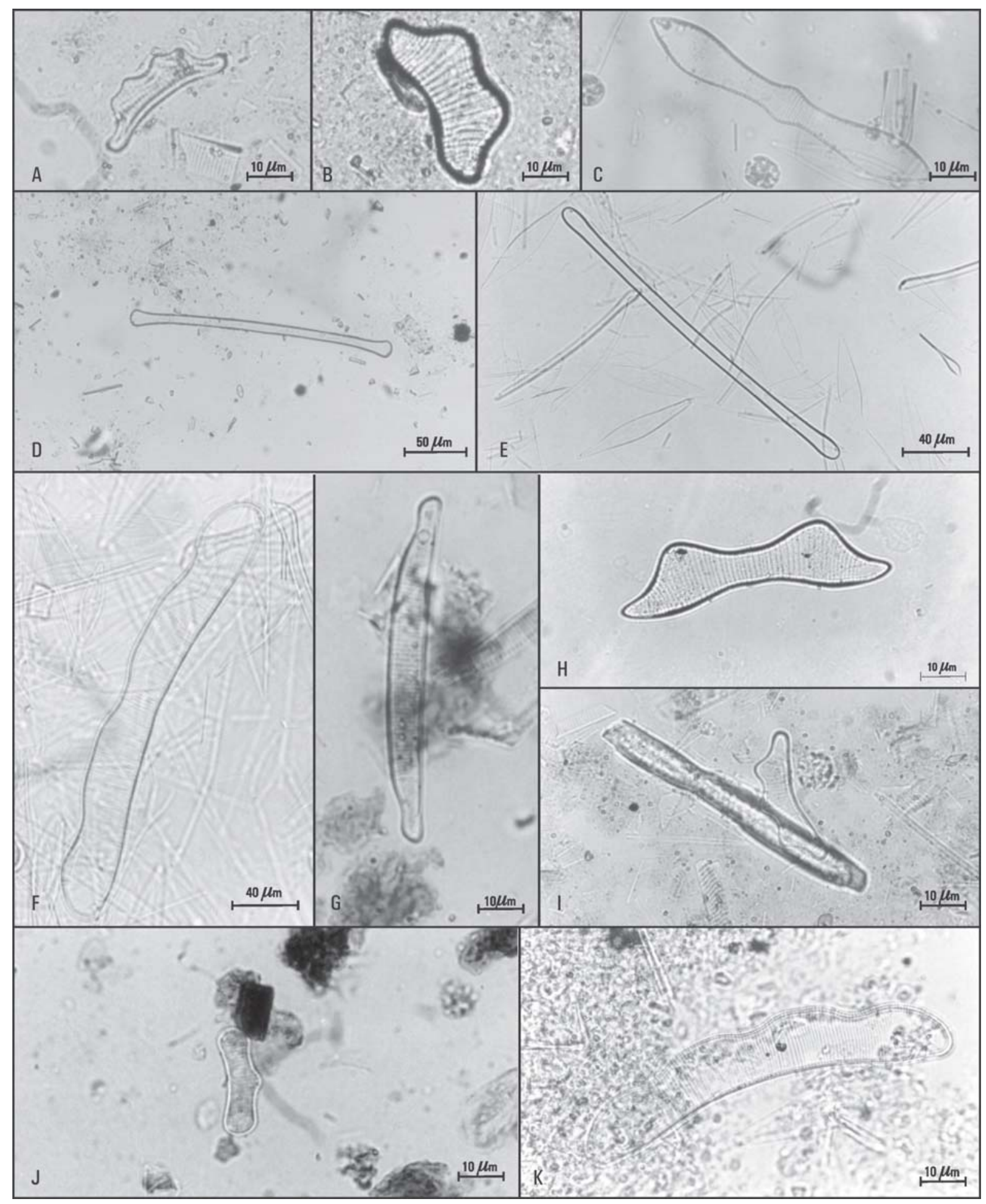

Figura 1. A. Eunotia camelus Ehrenberg. B. Eunotia didyma Grunow var. didyma. C. Eunotia didyma Grunow var. elongata Hustedt. D. Eunotia femoribus (Patr.) Hustedt. E. Eunotia flexuosa (Bréb.) Kützing. F. Eunotia major (W. Sm.) Rabh. var. emarginata A. Cleve. G. Eunotia pectinalis (Dill.). H. Eunotia didyma Grunow var. jungulata Freguelli. I. Eunotia trigibba Hustedt. J. Eunotia vumbae Choln. K. Eunotia zygodon Ehrenberg. 


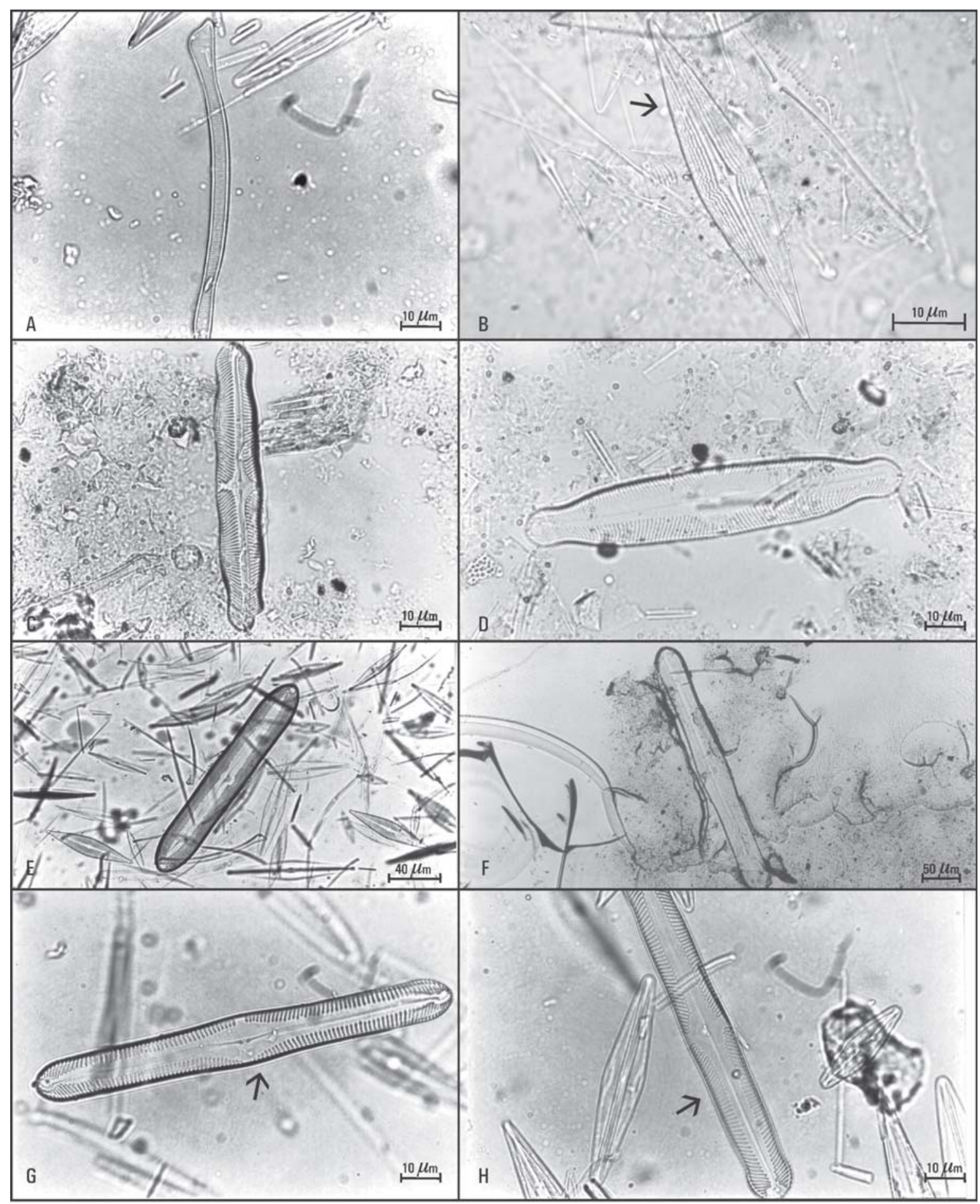

Figura 2. A. Actinella brasiliensis Grunow. B. Anomoeoneis serians (Bréb. Ex Kütz.) Cleve. C. Pinnularia abaujensis Pantocsek. D. Pinnularia biceps Gregory. E. Pinnularia maior (Kütz.) Rabh. F. Pinnularia nobilis Ehrenberg. G. Pinnularia stauroptera (Grun.) Rabenhorst. H. Pinnularia stomatophoroides Mayer. 


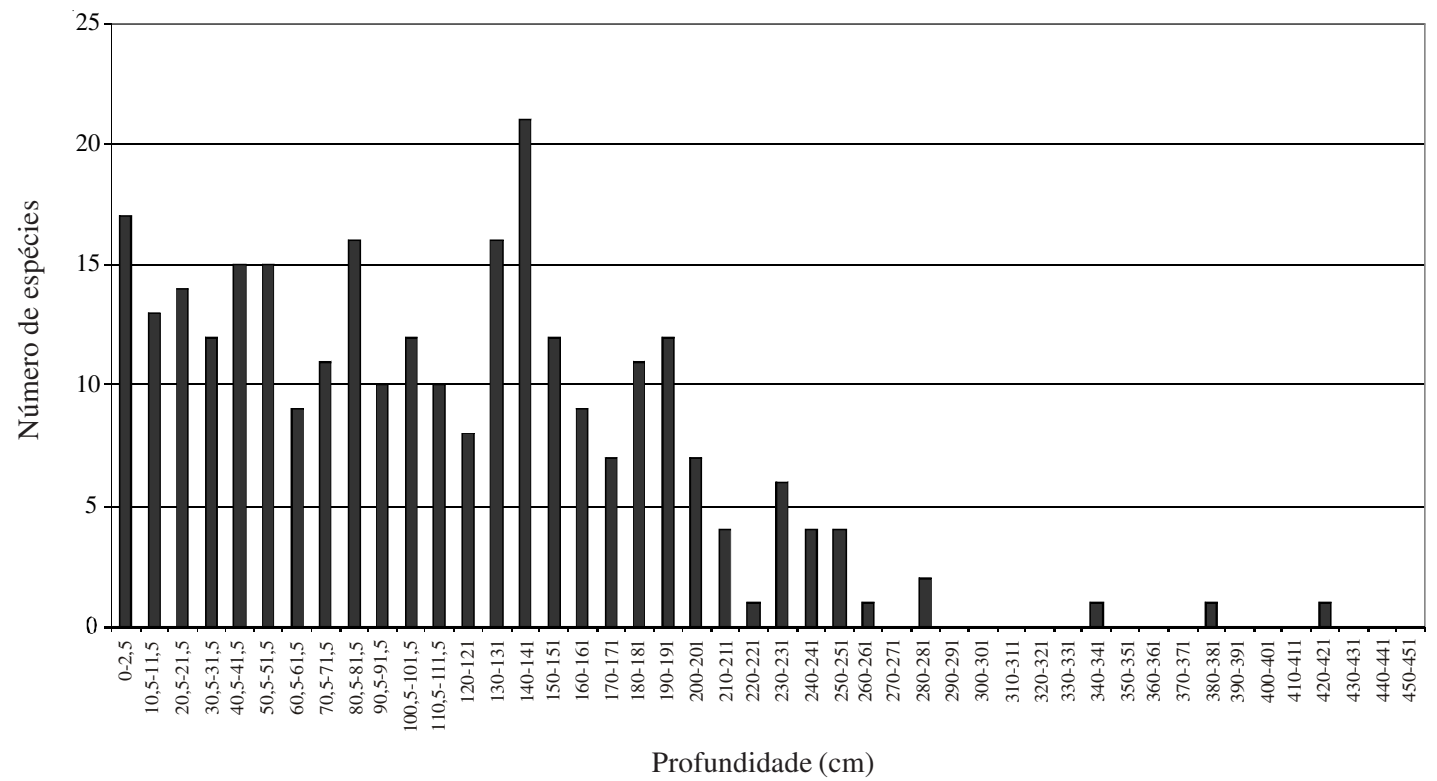

Figura 3. Número de espécies na coluna estratigráfica coletada em sedimentos do Quaternário de Dois Irmãos, Recife, PE, Brasil.

do predomínio dos elementos considerados oligoalóbios, os quais constituíram $80,6 \%$ da flora identificada (Tab. 2). A presença de espécies epífitas e bênticas
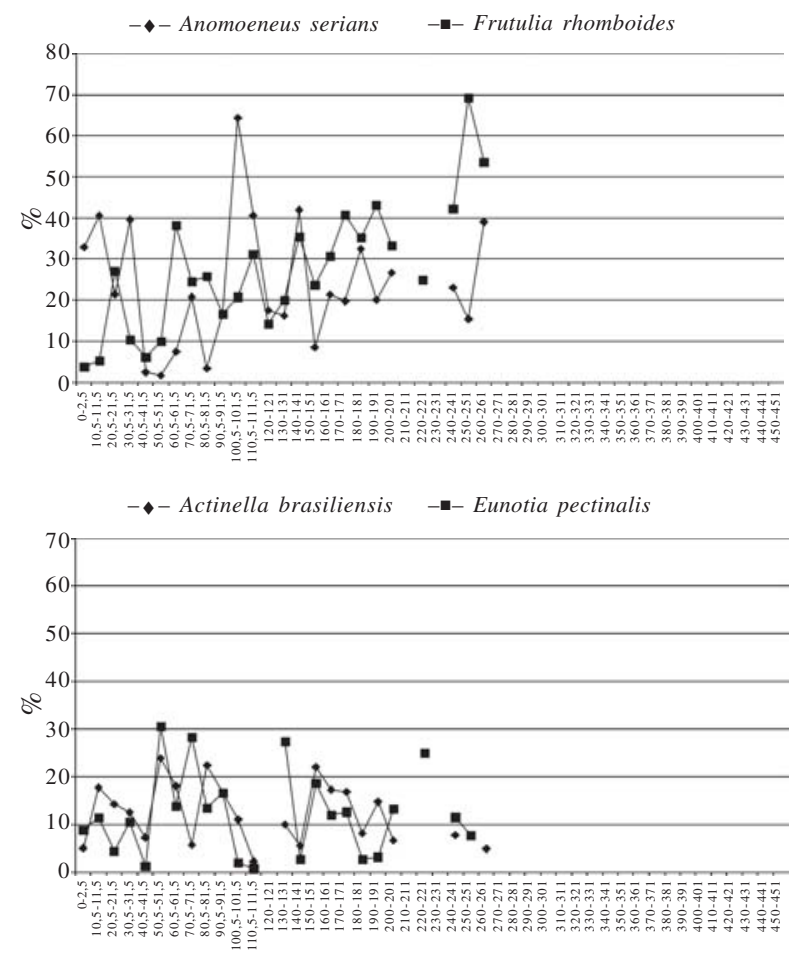

Figura 4. Abundância relativa das principais espécies de diatomáceas identificadas no testemunho de sondagem coletado em sedimentos do Quaternário de Dois Irmãos, Recife, PE, Brasil. sugerem a existência de um ambiente lacustre, de pequena profundidade, considerando-se o hábito litoral (principalmente epífito) da maioria das espécies identificadas em todas as camadas onde ocorreu à deposição. Esta constatação, também, pode confirmar, no referido ecossistema, a colonização de macrófitas aquáticas (angiospermas), elementos considerados substratos indispensáveis para a fixação de espécies de diatomáceas epífitas. Considere-se, ainda, que estudos recentes realizados em lagos localizados na área de sondagem (Vasconcelos et al. 1998), confirmaram a presença abundante de Eleocharis interstincta (Vahl.) Roemer \& Schult, macrófita aquática enraizada, densamente colonizada por diatomáceas, entre as quais destacaram-se Anomoeoneis serians e Frustulia rhomboides espécies, também, identificadas por estes autores como abundantes no material depositado. Este fato pode confirmar que os depósitos estudados foram provenientes de deposição atual, com menos de 7.000 A.P.

Para Silvestre \& Carvalho (1998), nos lagos que formam a bacia da Prata, localizada na área de Dois Irmãos, a flora planctônica é relativamente pobre e pouco abundante, devido à escassez de nutrientes no epilímnio, ao contrário da flora epífita que é bastante diversificada. Este fato, talvez, justifique a rara presença de espécies de diatomáceas planctônicas no material analisado, onde apenas três espécies foram identificadas, Aulacoseira granulata, Cyclotella meneghiniana e Skeletonema costatum, o que poderia 
Tabela 2. Categorias ecológicas das espécies diatomáceas identificadas na coluna estratigráfica coletada em sedimentos do Quaternário de Dois Irmãos, Recife, PE, Brasil.

\begin{tabular}{|c|c|c|c|}
\hline Categoria & $\mathrm{N}^{\mathrm{o}}$ táxons & $\%$ & Táxons \\
\hline \multicolumn{4}{|l|}{ Água doce } \\
\hline Oligoalóbia epífita & 16 & 44,5 & $\begin{array}{l}\text { Actinella brasiliensis, Eunotia camelus, Eunotia didyma var. didyma, } \\
\text { Eunotia didyma var. elongata, Eunotia didyma var. jungulata, Eunotia } \\
\text { flexuosa, Eunotia femoribus, Eunotia major var. emarginata, Eunotia } \\
\text { pectinalis, Eunotia trigibba, Eunotia vumbae, Eunotia zygodon, Frustulia } \\
\text { rhomboides, Gomphonema gracile, Neidium affine, Rhopalodia } \\
\text { musculus }\end{array}$ \\
\hline Oligoalóbia bentônica & 11 & 30,6 & $\begin{array}{l}\text { Anomoeoneis serians, Hantzschia amphyozys var. aequalis, Hantzschia } \\
\text { amphyozys var. capitata, Nitzschia sigmoidea, Nitzschia praelonga, } \\
\text { Pinnularia abaujensis, Pinnularia biceps, Pinnularia maior, Pinnularia } \\
\text { nobilis, Pinnularia stauroptera, Pinnularia stomatophoroides. }\end{array}$ \\
\hline Oligoalóbia planctônica & 2 & 5,5 & Aulacoseira granulata, Cyclotella meneghiniana. \\
\hline \multicolumn{4}{|l|}{ Marinha } \\
\hline Planctônica & 2 & 5,5 & Surirella heideni, Skeletonema costatum \\
\hline Planctônica epífita & 5 & 13,9 & $\begin{array}{l}\text { Cocconeis heteroidea, Cocconeis scutellum, Diploneis decipiens.Nitzschia } \\
\text { scalaris, Nitzschia sigma }\end{array}$ \\
\hline
\end{tabular}

atestar a falta de condições ecológicas para o desenvolvimento de elementos planctônicos.

Foi também constatada nos sedimentos a presença de espécies marinhas: Cocconeis heteroidea, Cocconeis scutellum, Diploneis decipiens,Nitzschia scalaris, Nitzschia sigma, Skeletonema costatum e Surirella heideni, além da presença de espículas de espongiários. Estas ocorrências, contudo, não representam, um indicativo de condições marinhas nas profundidades amostradas, visto que, a presença destas espécies foi considerada esporádica, tendo as mesmas apresentado valores inferiores a 3\%.

A baixa abundância de espécies em um ambiente particular pode ser causada, por exemplo, por um fator ambiental principal, uma combinação de fatores ambientais, competição com outras espécies ou alimento favorito de um predador (Snoeijs, 1999). No caso do perfil de Dois Irmãos, estudos aprofundados de reconstrução de paleoambientes são necessários para que se possa tecer comentários à respeito.

\section{Referências bibliográficas}

Bold, H.C. \& Wynne, M.J. 1985. Introduction to the algae. Structure and reproduction. $2^{\mathrm{a}}$ ed., New Jersey, PrenticeHall.

Cooper, S.R. Estuarine paleoenvironmental reconstructions using diatoms. 1999. Pp. 352-373. In: E.F. Stoerner \& J.P. Smol (eds.). The diatoms: applications for the environmental and sciences. Cambridge, University Press.
CPRM (Centro de Pesquisas de Recursos Minerais) 2001. Geologia e recursos minerais do Estado de Pernambuco. Recife.

Coutinho, R.Q.; Filho, M.F.L; Neto, J.B.S. \& Silva, E.P. 1998. Características climáticas e geomorfológicas e geotécnicas da reserva ecológica de Dois Irmãos. Pp. 21-49. In: I.C. Machado; A.V. Lopes \& K.C. Pôrto (orgs.). Reserva ecológica de Dois Irmãos: estudo em um remanescente de Mata Atlântica em área urbana (Recife - Pernambuco - Brasil). Recife, Secretaria de Meio Ambiente - SECTMA.

Eskinazi-Leça, E.; Silva-Cunha, M.G.G. \& Koening, M.L.; Chamixaes, C.C.B.; Passavante, J.Z.O. \& Feitosa, F.A.N. 1996. Microalgas. Pp. 61-68. In: E.V.S.B. Sampaio; S.V.J. Mayo \& M.R.V. Barbosa (eds.). Pesquisa Botânica Nordestina: progresso e perspectiva. Recife, Sociedade Botânica do Brasil, Seção Regional de Pernambuco.

Fürstenberger, C.B. 2001. Interpretações paleolimnológicas do Quaternário Recente a partir da análise da comunidade de diatomáceas (Bacillariophyceae) no sedimento do Rio Icatu, Município de Xique-Xique, estado da Bahia, Brasil. Tese de Doutorado. Universidade Paulista, Rio Claro.

Lobo, E. \& Leighton, G. 1986. Estruturas comunitárias de las fitocenosis planctônicas de los sistemas de desembocaduras de rios y esteros de la zona central de Chile. Revista Biologia Marina 22: 1-29.

Martin, L. \& Flexor, J.M. 1995. Vibro testemunhador leve: construção, utilização e possibilidades. Revista do Instituto Geológico 16: 59-66.

Mateucci, S.D. \& Colma, A. 1982. La metodologia para el estudo de la vegetación. Colleción de monografias científicas. Série Biologia 22: 1-168. 
Moreira, I.M.V. 1975. Contribuição ao estudo das Bacillariophyceae (Diatomáceas) em diatomitos brasileiros. Curitiba, Acta de Biologia do Paraná 4(3-4): 135-198.

Moreira Filho, H.; Eskinazi-Leça, E.; Valente Moreira, I.M. \& Cunha, J.A. 1999. Avaliação taxonômica e ecológica das diatomáceas (Chrysophyta - Bacillariophyceae) marinhas e estuarinas nos estados de Pernambuco, Paraíba, Rio Grande do Norte, Ceará, Piauí, Maranhão, Pará e Amapá, Brasil. Trabalhos Oceanográficos da Universidade Federal de Pernambuco 27(1): 55-90.

Moreno, J.L.; Licea, S. \& Santoyo, H. 1996. Diatomeas del Golfo de California. La Paz: Universidad Autónoma de Baja California Sur.

Moro, R.S. \& Bicudo, C.E.M. 1998. Flutuações climáticas mo Pleistoceno tardio e Holoceno na lagoa Dourada, Estado do Paraná, Brasil. Acta Limnologica Brasiliensis 10(2): 47-58.

Müller-Melchers, F.C. \& Ferrando, H.J. 1956. Técnica para el estudio de las diatomeas. Boletim do Instituto Oceanográfico de São Paulo 7(1-2): 1- 273.

Ruiz-Moreno, J.L. \& Carreño, A.L. 1994. Diatom biostratigraphy of Bahia Asunción, Baja Califórnia Sur, México. Revista Mexicana de Ciências Geológicas 11(2): 243-252.

Silvestre, A.N. \& Carvalho, P.V.B.C. 1998. Bacia do Prata: aspectos qualitativos da água. Pp. 51-83. In: I.C. Machado; A.V. Lopes \& K.C. Pôrto (orgs.). Reserva ecológica de Dois Irmãos: estudo em um remanescente de Mata Atlântica em área urbana (Recife - Pernambuco Brasil). Recife, Secretaria de Meio Ambiente - SECTMA.
Simonsen, R. 1979. The diatom system: ideas on phylogeny. Bacillaria 2: 9-71.

Souza, H.C.A. \& Abreu, S.F. 1939. Diatomito do Nordeste. Rio de Janeiro. Boletim Departamento Nacional de Produção Mineral 33: 1-58.

Snoeijs, P. 1999. Diatoms and environmental change in brackish waters. Pp. 298-333. In: E.F Stoerner \& J.P. Smol. (eds.). The diatoms: applications for the enviromental and earth sciences. Cambridge, University Press.

Suguio, K. 1999. Geologia do Quaternário e mudanças ambientais: (passado + presente $=$ futuro). São Paulo, Paulo's Comunicação e Artes Gráficas.

Vasconcelos, A.C.C.; Eskinazi-Leça, E.; Passavante, J.Z.O. \& Lopes-Chamixaes, C.B.C.B. 1998. Algas perifíticas (epífitas) dos açudes do Vale do Prata (Reserva Ecológica de Dois Irmãos). Pp. 65-83. In: I.C. Machado; A.V. Lopes \& K.C. Pôrto (orgs.). Reserva ecológica de Dois Irmãos: estudo em um remanescente de Mata Atlântica em área urbana (Recife - Pernambuco Brasil). Recife, Secretaria de Meio Ambiente SECTMA.

Yim, W.W. S. \& Li, J. 2000. Diatom preservation in an inner continental shelf borehole from the South China Sea. Journal of Asian Earth Sciences 18(4): 471-488.

Zalat, A.A. 1995. Diatoms from the Quaternary sediments of the Nile Delta, Egypt, and their palaeoecological significance. Journal of African Earth Sciences 20(2): 133-150. 